\title{
The Cutting Force Prediction of Standard Twist Drill
}

\author{
Pengpeng Zhang \\ Tianjin Long March Launch Vehicle Manufacturing Co. \\ Ltd \\ Tianjin, China
}

\author{
HouJun Qi \\ Mechanical Engineering, Tianjin University of \\ Technology and Education, Tianjin Key Lab of High \\ Speed Cutting and Precision Machining, \\ Tianjin, China \\ qihoujun@163.com
}

\author{
Hui Yao \\ Tianjin Long March Launch Vehicle Manufacturing Co. \\ Ltd \\ Tianjin, China \\ YiRong Zhang \\ Mechanical Engineering, Tianjin University of \\ Technology and Education, Tianjin Key Lab of High \\ Speed Cutting and Precision Machining, \\ Tianjin, China \\ zhangyirong3399@163.com
}

\begin{abstract}
Based on the research of the standard twist drill geometry parameters, the cutting part of the structure of the geometric parameters are expressed as a fuction of radius, the chisel edge and the main cutting edge geometric parameters of the mathematical model are established. Drilling force is one of the most important parameters in the process of drilling, it directly affects the size of the drilling quality, but the geometry of a drill make the drilling process complicated. Under certain spindle speed and feed rate, apply MATLAB software simulation to drilling force model, achieve the prediction of the dynamic of drilling force and torque of the forecast.
\end{abstract}

Keywords-Twist drill; geometrical parameter; Mechanistic model; MATLAB; Prediction.

\section{INTRODUCTION}

Drilling force is one of the most important parameters in the process of drilling, it directly affects the size of the drilling quality, but the geometry of a drill make the drilling process complicated. Since the cutting geometry parameters of every point on the cutting edges are changing, it could cause drilling force is too large, the bit life shortened, and the problem of drill bit break and the surface quality and dimensional accuracy has great influence on account of improper selection of processing parameters in the process of drilling. The complexity of drilling process is to need to do a lot of experiments to choose the optimization of processing parameters, not only cost a lot of time and cost, and the result is often not optimal.

Earlier studies have focused on establishing empirical torque and axial force model, the modeling method is through the drilling experiment experience deduced formula of cutting force. Lee $\mathrm{S} \mathrm{W}$ and Fuh KH ${ }^{[1]}$ established the group of diamond cutting force model on the basis of work cutting angle. Huang HT ${ }^{[1]}$ get the method of using ordinary twist drill mechanics model of prediction of drilling axial force and torque. $J$ Strenkowski ${ }^{[2]}$ at the university of Michigan established the oblique cutting model of drilling process, the drill tip as a series of oblique cutting along the cutting edge direction unit of cutting tool, and to bring the bevel cutting finite element model is applied to the drilling axial force and torque of the analysis and research. Kawaji $\mathrm{S}^{[3]}$ put forward a kind of neural network model to estimate and control method of drilling axial force. Strenkowski JS and others using finite element technology put forward the forecast method of twist drill axial force and torque, with a eulerian finite element model to simulate unit tool cutting force of cutting edge $\mathrm{e}^{[4,5]}$.

This paper studies the processing of high speed drilling, establish dynamic drilling force and torque model, get the graph by simulation with MATLAB software.

\section{THE GEOMETRIC PARAMETERS OF THE STANDARD TWIST DRILL}

The sword of twist drill structure are shown in Fig.1. The significant parameters that describe the geometry of a conical point drill include the drill diameter( $D=2 R$ ),point angle( $\left.2 \varphi=119^{\circ}\right)$,helix angle $\left(\beta_{0}\right)$,web thickness $\left(d_{c}=2 w, w\right.$ is chisel edge width), and chisel edge angle $(\psi)$.

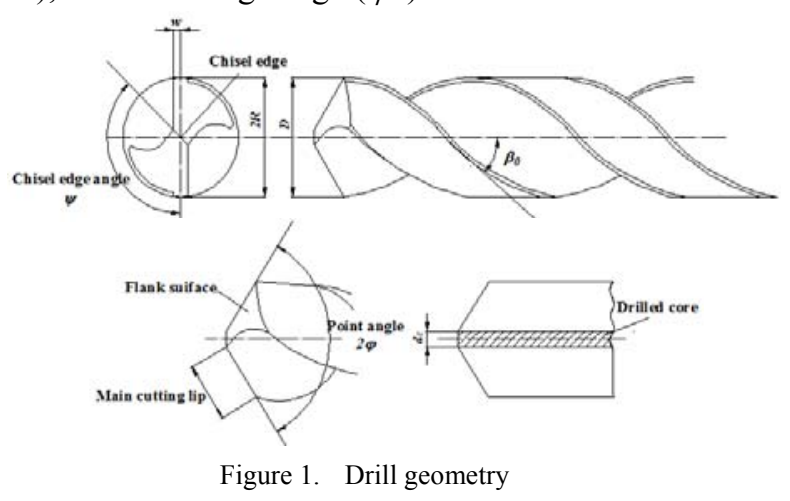


Bit of main cutting edge geometry relationship is more complex, in order to establish the forecast model of drilling force must identify the bit cutting geometry parameters: helix angle, inclination angle, tool cutting edge angle, normal rake angle.

(i)Helix angle and inclination angle

The helix angle at any point on the main cutting edge is determined by the helix angle of cutting edge the edge points:

$$
\tan \beta=\frac{r}{R} \tan \beta_{0}
$$

As seen in (1), which is the helix angle at the outside radius is the largest, that is equal to $\beta_{0}$. The more near the center of the bit, the smaller helix angle. The helix angle is actually to the rake angle of the drill tip $\left(\beta=\gamma_{f}\right)$. Therefore the bigger the helix angle, the greater the rake angle of the drill tip, and the greater the bit more sharp. The helix angle of Standard twist drill is between $18^{\circ} \sim 30^{\circ}$ commonly.

The main cutting edges are as a result of the twist drill by bit axis, forming a inclination angle, it is the angle between the main cutting edge and the base surface between the cutting plane. Because the points on the main cutting edge of the cutting plane of the base surface at different positions, thus the inclination angle is varied. A base end surface with a cutting edge inclination angle of the main cutting edge on the main cutting edge twist angle at any point on the end face of the projection, and its effect is negative. It is a function of the radius in (2):

$$
\sin \lambda_{s t}=-\frac{d_{c}}{2 r}
$$

And the end face of the edge cutting edge inclination angle angle on the relationship between the main cutting edge twist at any point is:

$$
\sin \lambda_{s}=\sin \lambda_{s t} \sin \varphi
$$

From the formula (2) and (3) it can be seen, the inclination angle of the cutting edge with only point angle, thickness, and the selected point terrible radius, that is, when determining the amount of time these three drill, drill variation of cutting edge inclination angle $\lambda_{s}$ is determined by the radius of the selected point $r$.For the standard twist drill of drill diameter( $D=18 \mathrm{~mm})$,drill core diameter $\left(d_{c}=2 w=3 \mathrm{~mm}\right)$, point angle $\left(2 \varphi=118^{\circ}\right)$, the law changes along the cutting edge of its cutting edge inclination angle $\lambda_{s}$ are shown in Fig.2.

(ii)Tool cutting edge angle ${ }^{\kappa_{r}}$ and normal rake angle $\gamma_{n}$

The tool cutting angle is the angle between the drill main cutting edge in the basal plane of the projection and the feed direction. The tool cutting angle at any point along the cutting lips of the drill is as follows:

$$
\tan \kappa_{r}=\tan \varphi \cos \lambda_{s t}
$$
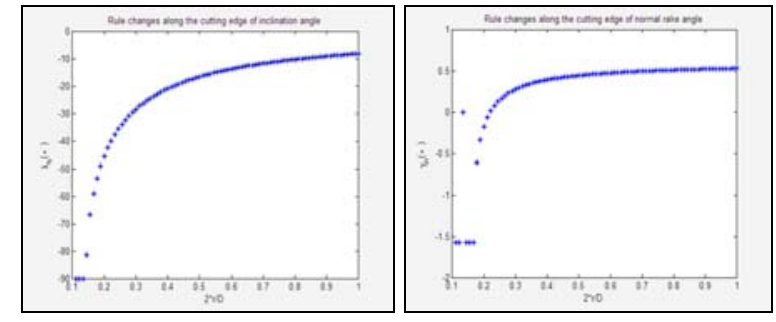

Figure 2. Variation of inclination angle $\lambda_{s}$ and tool cutting edge

$$
\text { angle } \kappa_{r} \text { with the its cutting edge }
$$

As seen from formula (4), the closer drill core, the tool cutting angle smaller.

The cutting edge of the drill at any point forward the relationship between the normal rake angle $\gamma_{n}$ and feed rake angle ${ }^{\gamma_{f}}$ as follows:

$$
\tan \gamma_{n}=\tan \gamma_{f} \cos \lambda_{s}
$$

The formula (1) (3) into Equation (5), finishing available:

$$
\tan \gamma_{n}(r)=\frac{2 \tan \beta_{0}}{D \sin \varphi}\left(\sqrt{r^{2}-w^{2}}+\frac{w^{2} \cos ^{2} \varphi}{\sqrt{r^{2}-w^{2}}}\right)-\frac{w \cos \varphi}{\sqrt{r^{2}-w^{2}}}
$$

For the standard twist drill of helix angle $\beta=30^{\circ}$, point angle $2 \varphi=118^{\circ}$, Fig. 2 shows the variation in the normal rake angle with the radial distance along the cutting lips. As seen in Fig.2, the normal rake angle varies considerably along the cutting lips, from negative value toward the chisel edge and to large positive values near the outer radius of the drill.

\section{MECHANISTIC FORCE MODEL FORMULATION}

Axial thrust force and the lateral force during the drilling come from the chisel edge, the cutting lips and the secondary edge three parts. With the rate $f_{r}$ to feed, the cutting lips cut with a constant chip thickness $(h)$ to remove material. Axial force to advance the drill bit, drill the torque exerted on the role of the spindle in overcoming drilling torque. By a large number of experimental studies that, the sum of the axial force, the cutting lips account for $40 \%$, the chisel edge is approximately 1.4 times the cutting lips, and the minimum proportion of secondary edge. In sum torque, the cutting lips account for $60 \%$, mainly chisel edge about half of the cutting lips, and the rest from the secondary edge.

(i)Force model formulation for chiesl edge

Chisel edge do not performe the cutting, it's just by squeezing mechanism to the side to squeeze the material. However, the mechanism of horizontal geometry and blade squeeze quite complex and require detailed experience on a variety of geometric modeling and experimental calibration factors ${ }^{[6]}$. Although people have done a lot of research, but still cannot predict more accurate drilling force. If this extrusion process is simplified as hardness testing, the axial thrust force acting on the chisel edge can be expressed as:

$$
F_{z . i}=A_{c h} H_{B}
$$




$$
A_{c h}=\frac{2 w f_{r}}{\sin (\pi-\psi) \cos \varphi}
$$

Where HB is brinell hardness of workpiece material, and $A_{c h}$ is the instantaneous extrusion area of chisel edge, which is equal to the product of chisel edge length $[2 w / \sin (\pi-\psi)]$ and contact length of material to be extruded and the cutting chips $\left[f_{r} /(2 \cos \varphi)\right]$.

The chisel edge is approximately reduced to a simple squeeze for accurate drilling force study is unreliable. In practical consideration, the cutting force on the chisel edge can be calculated by the force of the proportion of the force on the cutting chips of the total cutting forces.

(ii)Force model formulation for the cutting lips

During the cutting process, the cutting velocity, as well as the inclination angle and normal rake angle, varies with the radial distance $(r)$ along the cutting lips of the drill. The tangential cutting velocity is a linear function of the radial distance and increases outward. For the radial distance $(r)$, the governing equations for the tangential cutting velocity $(v)$ is as follows:

$$
v(r)=\frac{2 \pi r N}{60}
$$

In order to study the forces of the cutting lips, first it need to consider participating in the length of the cutting portion of the cutting lips. Because it is increases with time until the cutting lips full access to the workpiece, it can be represented by the axial distance of the drill cutting lips to the point:

$$
r(t)=\left\{\begin{array}{l}
{\left[r_{s}^{2}+2 \Delta\left(r_{s}^{2}-w^{2}\right)^{\frac{1}{2}}+\Delta^{2}\right]^{\frac{1}{2}}, r<D / 2} \\
D / 2, r \geq D / 2
\end{array}\right.
$$

In the formula, $\Delta=t \cdot f_{r} \cdot n / 60 \cdot \tan \varphi . r_{s}$ is the radius of the prepared hole, under normal circumstances: $\frac{b_{\psi}}{2} \leq r_{s} \leq R$; when there is no pre-drilling, $r_{s}$ can be regarded as half the length of the chisel edge $\left(r_{s}=b_{\psi} / 2\right)$. The length of the chisel edge is $b_{\psi}=d_{c} / \sin \psi$.

Each cutting lip of the drill was divided into a number of elements which the thickness is $t_{c}$. when the feed rate was $f_{r}$, the uncut chip area of each element on the cutting lip can be represented as $d A_{c}$. For each element, the cutting force (the normal $\left(d F_{n}\right)$ and friction force $\left(d F_{f}\right)$ ) acting on the tool are assumed to be proportional to the chip load. The chip load is the projected area of the shear plane measured in a plane normal to the cutting velocity vector.

$$
\begin{aligned}
& d A_{c}=f_{r} \cos \lambda_{s} \sin \varphi d r / 4 \\
& d F_{n}=k_{n} d A_{c} \\
& d F_{f}=k_{f} d A_{c}
\end{aligned}
$$

The specific normal pressure $\left(k_{n}\right)$ and the specific friction pressure $\left(k_{f}\right)$ are dependent on the uncut chip thickness $\left(t_{c}\right)$, normal rake angle $\left(\lambda_{n}\right)$, and cutting velocity $(v)$.Numerous experiments show the specific normal and friction pressures can be described as linear expressions in the logarithmic domain, as below ${ }^{[7]}$ :

$$
\begin{aligned}
& \ln k_{n}=x_{0}+x_{1} \ln t_{c}+x_{2} \ln v+x_{3} \ln \left(1-\sin \gamma_{n}\right) \\
& \ln k_{f}=y_{0}+y_{1} \ln t_{c}+y_{2} \ln v+y_{3} \ln \left(1-\sin \gamma_{n}\right)
\end{aligned}
$$

Where the coefficient $x_{i}, y_{i}$ can be obtained by multiple linear regression of test data are shown in Table1.The regression results (Table 1) were given in the literature by Chandrasekharan.

TABLE I. COEFFICIENTS OF SPECIFIC AND FRICTION PRESSURES OBTAINED FROM CALIBRATION EXPERIMENT

\begin{tabular}{lllll}
\hline & 0 & 1 & 2 & 3 \\
\hline$x_{i}$ & 5.549 & -0.359 & -0.097 & 1.338 \\
$y_{i}$ & 3.734 & -0.679 & -0.064 & -0.217
\end{tabular}

Three force components, including the cutting force $d F_{a}$, thrust force ${ }^{d F_{t}}$, and lateral force $d F_{r}$,can be related to the elemental normal and friction forces, as shown below:

$$
\left\{\begin{array}{l}
d F_{a} \\
d F_{t} \\
d F_{r}
\end{array}\right\}=\left[\begin{array}{ll}
\cos \eta_{c} \cos \gamma_{n} & -\sin \gamma_{n} \\
\sin \eta_{c} \sin \lambda+\cos \eta_{c} \cos \lambda \sin \gamma_{n} & \cos \lambda \sin \gamma_{n} \\
\cos \eta_{c} \sin \lambda \sin \gamma_{n}-\sin \eta_{c} \cos \lambda & \sin \lambda \cos \gamma_{n}
\end{array}\right] \times\left\{\begin{array}{l}
d F_{f} \\
d F_{n}
\end{array}\right\}
$$

Where $\eta_{c}$ is the chip flow angle, which will be set here to be equal to the inclination angle $\lambda_{s}$ for calculation convenience. Then, the elemental axial thrust force $d F_{z}$ and torque $d M_{z}$ can be calculated using the three force components, as shown below:

$$
\left\{\begin{array}{l}
d F_{z} \\
d M_{z}
\end{array}\right\}=\left[\begin{array}{ccc}
\frac{\cos \beta \sin \varphi}{\cos \lambda_{s}} & 0 & -\frac{\cos \varphi}{\cos \lambda_{s}} \\
0 & r & 0
\end{array}\right] \times\left\{\begin{array}{l}
d F_{t} \\
d F_{a} \\
d F_{r}
\end{array}\right\}
$$

The magnitude of the total drilling axial thrust force $F_{z}$ and torque $M_{z}$ from the two cutting lips can be obtained by summing the forces at all the elements for both cutting lips as:

$$
\left\{\begin{array}{l}
F_{z}=\int_{r_{s}}^{R} 2 d F_{z} \\
M_{z}=\int_{r_{s}}^{R} 2 d M_{z}
\end{array}\right.
$$

According to the cutting force generated by the cutting lips accounted for the proportion of the total axial force, a total size of axial force and torque of the drill bit for drilling are as follow:

$$
\left\{\begin{array}{l}
F_{Z}=2.5 \int_{r_{s}}^{R} 2 d F_{z} \\
M_{Z}=1.67 \int_{r_{s}}^{R} 2 d M_{z}
\end{array}\right.
$$

\section{SIMULATION RESULTS}

Based on the calculation of given bit drilling force and torque in the process of drilling model, using Matlab software to the simulation predictions. Set the drill tip diameter of $6 \mathrm{~mm}$, speed $400 \mathrm{r} / \mathrm{mm}$, feed $0.1 \mathrm{~mm} / \mathrm{r}$, Bit of axial force and torque simulation image below. Simulation 
image intuitively shows that with the increase of twist drill diameter, the axial force and torque increased.

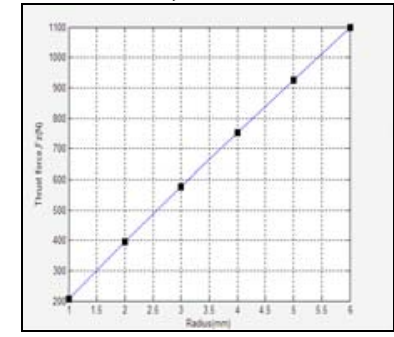

(a) $n=400 \mathrm{rpm}, f_{\mathrm{r}}=0.1 \mathrm{~mm} / \mathrm{rev}$

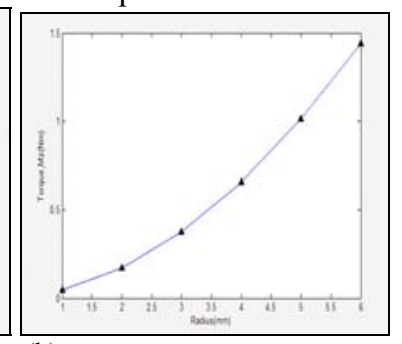

(b) $n=400 \mathrm{rpm}, f_{r}=0.1 \mathrm{~mm} / \mathrm{rev}$
Figure 3. Thrust force (a) and torque (b) model predictions

\section{CONCLUSIONS}

By analyzing the geometric parameters of the standard twist drill, a mechanistic force model for predicting the thrust force and torque when drilling was established. The simulation need to select a certain cutting parameters, and take advantage of Matlab to forecast axial force and torque of drilling process. Rely on the drilling force that predicted under the specific processing conditions to select the best geometric parameters of the bit and drilling process of processing parameters to improve the quality of drilling.

\section{ACKNOWLEDGMENT}

This work was supported by "High-End CNC Machine Tools and Basic Manufacturing Equipments" Major National Science and Technology Project (2012ZX04012031), Key Projects in the Tianjin Science and Technology Pillar Program (14ZCZDGX00002), Science and Technology Development Fund Project of Tianjin Higher Education Institutions of China(2008ZD17 and 20130408),
Innovation Team Training Plan of Tianjin Universities and colleges (TD12-5043) and Tianjin University Technology \& Education Foundation ( KJ10-03).

\section{REFERENCES}

[1] Bao Luo.Studies and drilling process is complex Helical Drill Point Drilling Force finite element simulation [D], Northeastern University:2009

[2] J S Strenkowski,C C Hsieh,A J Shih,An analytical finite ele-ment technique for predicting thrust force and torque in drilling.International Journal ofMachineTools\&Manufacture , 44, 2004, pp.1413 1421

[3] Pengfei Yan. Stainless steel drilling finite element simulation and experimental study[D], Northeastern University:2010

[4] Fanglai Zhu,Zhongxin Ye,Yurong Chen. Drilling Force mathematical model[J], Hubei Institute of Technology,1997.9:7073

[5] Liangshan Xiong,Hanmin Shi,Yongjie Chen. History and current situation and development trend of the drill bit and drilling studies $[\mathrm{J}], 2005,39(8): 11-14$

[6] E.J.A.Armarego , D.Pramanik , A.J.R.Smith , and Whitfield R.C.Forces and Power in Drilling-Computer Aided Predictions.Journal of Production Engineering, 6:149 174,1983.

[7] Chandrasekharan,V.1996.A Model to Predict the ThreeDimensional Cutting Force System for Drilling with Arbitrary Point Geometry.University of Illinois at Urbana-Champaign,Ann Arbor,pp. 178

[8] Lim, T.C., Singh, R., 1990. Vibration transmission throught rolling element bearings,Part I to Part III. Journal of Sound and Vibrations 139 (179-199), 201-225.

[9] Lim, T.C., Singh, R., 1990. Vibration transmission throught rolling element bearings,Part I to Part III. Journal of Sound and Vibrations 139 (179-199), 201-225.

[10] Bayly, P.V., Metzler, S.A., 2001. Theory of torsional chatter in twist drills: model,stability analysis and composition to test. Journal of Manufacturing Science andEngineering 123, 552-561. 